\title{
Anesthetic management for the insertion of a self-expandable metallic tracheal stent under venovenous extracorporeal membrane oxygenation
}

\author{
Sang Hyun Hong, Young Eun Moon, Se Rin Lee, Sun Jin Cho, and Ou Kyoung Kwon
}

Department of Anesthesiology and Pain Medicine, Seoul St. Mary's Hospital, College of Medicine, The Catholic University of Korea, Seoul, Korea

Airway stents can provide prolonged palliation from endoluminal malignant tumors when the complete tumor resection is not possible [1]. General anesthesia is frequently used to facilitate the procedure by establishing a quiet operative field [2]. The present report describes the anesthetic management of tracheal stent insertion by rigid bronchoscopy using venovenous extracorporeal membrane oxygenation (VV ECMO).

A 69-year-old man, $175.6 \mathrm{~cm}$ in height and $64.9 \mathrm{~kg}$ in weight, who had undergone total gastrectomy for gastric cancer 9 years earlier, was scheduled for tracheal stent insertion using a rigid bronchoscope. One year before the procedure, he had developed lymph node metastasis at the upper mediastinum, between the trachea and esophagus. The mass had continued to grow into the trachea and aggravated dyspnea (Fig. 1A). The stenotic segment had a diameter of $3 \mathrm{~mm}$ and a length of 2.6 $\mathrm{cm}$, based on the CT scan. A pulmonary function test revealed severe airway obstruction, as indicated by a forced expiratory volume at $1 \mathrm{~s} /$ forced vital capacity ratio (FEV1/FVC) of $22 \%$.

The procedure was performed in the operating room under general anesthesia. Upon arrival in the operating room, the patient demonstrated a slight retraction of the intercostal muscles, but endured a supine position. With sufficient preoxygenation, anesthesia was carefully induced with propofol and remifentanil using target-controlled infusion. After we confirmed that anesthetic induction had not compromised manual ventilation via a facial mask, tracheal intubation was facilitated with rocuronium. A reinforced endotracheal tube with an inner diameter of $7.0 \mathrm{~mm}$ was advanced into the
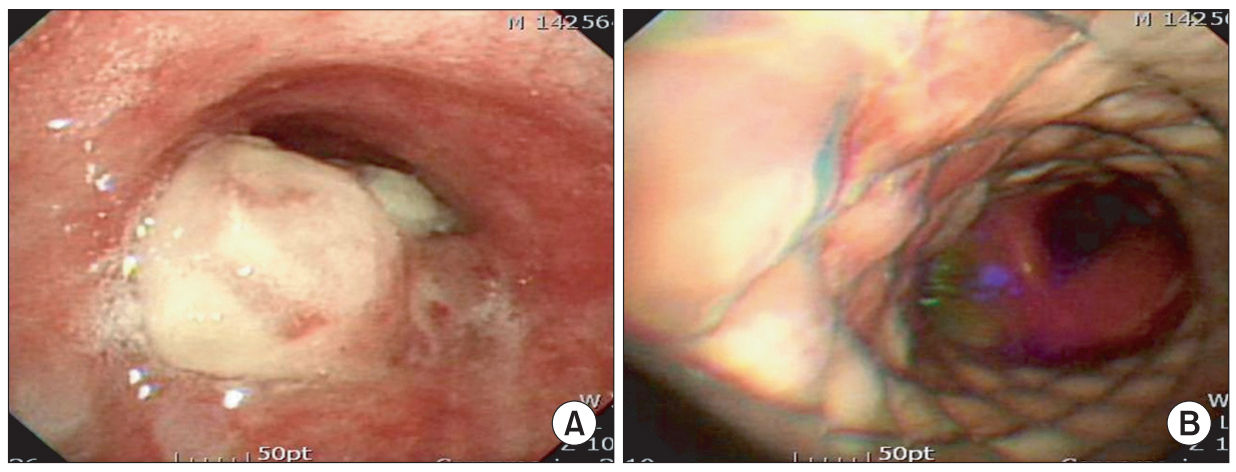

Fig. 1. (A) Preoperative bronchoscopy reveals a metastatic mass infiltrating the trachea. (B) Postoperative bronchoscopy shows that the tracheal lumen is widened by a covered self-expandable metallic tracheal stent.

Corresponding author: Ou Kyoung Kwon, M.D., Department of Anesthesiology and Pain Medicine, Seoul St. Mary's Hospital, College of Medicine, The Catholic University of Korea, 505, Banpo-dong, Seocho-gu, Seoul 137-701, Korea. Tel: 82-2-2258-2236, Fax: 82-2-537-1951, E-mail:kwon208@catholic.ac.kr

(c) This is an open-access article distributed under the terms of the Creative Commons Attribution Non-Commercial License (http:// creativecommons.org/licenses/by-nc/3.0/), which permits unrestricted non-commercial use, distribution, and reproduction in any medium, provided the original work is properly cited. 
patient's trachea and placed with the upper margin of the cuff located just below the patient's vocal cords. Manual ventilation led to normal inflation of both chest walls and a normal capnograph. An arterial blood gas analysis (ABGA) showed adequate ventilation and oxygenation.

After anesthetic induction, VV ECMO was established. Cannulation was performed with a $24 \mathrm{Fr}$ percutaneous venous cannula at the right femoral vein for outflow and with an $18 \mathrm{Fr}$ percutaneous venous cannula at the left internal jugular vein for inflow. An extracorporeal life support system (Capiox EBS, Terumo Inc., Tokyo, Japan) was primed with $1 \mathrm{~L}$ of normal saline. Systemic anticoagulation was attained using intravenous heparin to achieve an activated clotting time between 180-200 s. The perfusion flow rate was gradually increased to $3.0 \mathrm{~L} / \mathrm{min}$, causing an abrupt decrease in blood pressure. The patient's blood pressure (BP) dropped to $65 / 40 \mathrm{mmHg}$, with a heart rate (HR) of 120 beats/min. Ephedrine $10 \mathrm{mg}$ was administered, followed by epinephrine $10 \mu \mathrm{g} .500 \mathrm{ml}$ of colloid solution was infused over $10 \mathrm{~min}$. After resuscitation, the patient's vital signs were stable, and the procedure was performed.

A rigid bronchoscope was advanced into the trachea after removing the endotracheal tube. The stent delivery catheter was then inserted into the stenotic segment, and a covered self-expandable metallic tracheal stent, with a length of $6 \mathrm{~cm}$ and diameter of $18 \mathrm{~mm}$ (Hercules Airway, S\&G Biotech Inc., Seongnam, Korea), was deployed. After the delivery catheter was removed, the trachea was re-intubated, and the endotracheal tube was positioned similarly to the first intubation. Throughout the procedure, global oxygenation assessed by serial ABGAs was adequate with ECMO augmented by intermittent positive pressure ventilation via a side port of the rigid bronchoscope. Perfusion flow of the ECMO system was maintained at $3.0-4.0 \mathrm{~L} / \mathrm{min}$, with oxygen flow around $4.0 \mathrm{~L} /$ min at $\mathrm{FiO}_{2}$ of 1.0.

When the procedure was complete, the patient was transferred to the intensive care unit. To wean the patient from ECMO, the oxygen flow of the ECMO system was gradually decreased from $4.0 \mathrm{~L} / \mathrm{min}$ to $0 \mathrm{~L} / \mathrm{min}$. After ensuring that removal of the oxygen flow would not result in hypoxia, the perfusion flow was decreased and stopped, and the cannulas were removed. The tracheal stent relieved the upper airway obstruction on a postoperative pulmonary function test, as indicated by an FEV1/FVC of $61 \%$ (Fig. 1B).

The potential for critical airway obstruction is an important concern during tracheal stent insertion. Thus, various ventilation modes including maintenance of spontaneous ventilation, intermittent positive pressure ventilation via a side port of a rigid bronchoscope, or low- or high-frequency jet ventilation have been introduced [2]. Neither spontaneous ventilation nor intermittent positive pressure ventilation via the ventilating port was feasible in the present case because the stenosis of the trachea by the metastatic mass was so severe that the passage of a delivering catheter into the stenotic segment could have caused complete airway obstruction. Low-frequency jet ventilation from above the stenotic segment through a rigid bronchoscope was also not possible because the flow would not reach the lower respiratory tract beyond the stenotic segment during stent deployment. A high-frequency jet ventilator was not available in our hospital. Distal tracheal intubation was not possible because the mass was located intrathoracically. Therefore, the decision was made to establish VV ECMO prior to the tracheal stent insertion.

There are several considerations when using ECMO for airway management in a patient undergoing airway stent insertion. First, it should be determined preoperatively whether ECMO is to be initiated before or after the administration of anesthesia because anesthetic induction can induce catastrophic airway obstruction. Second, standby ECMO may be a sensible choice in some cases because instituting ECMO is associated with coagulopathy, infection, and cannulation site complications [3]. Catheter insertion without initiating extracorporeal blood flow could reduce the time required to establish ECMO. Third, intermittent positive pressure ventilation via a side port of a rigid bronchoscope may be necessary to maintain oxygenation. In a patient with respiratory failure, a target arterial oxygen saturation achieved by VV ECMO is between $85-95 \%$ [4]. Applying ECMO in a patient receiving an airway stent allows the functioning lungs to be used for intermittent positive pressure ventilation, which can optimize oxygenation.

VV ECMO is considered a valuable option during tracheal stent insertion that is expected to cause critical airway obstruction. For optimal ventilatory support, a strategy for ventilation and anesthesia should be established prior to the procedure, according to the degree of tracheal obstruction, the nature of the mass, and the method being used.

\section{References}

1. Gaafar AH, Shaaban AY, Elhadidi MS. The use of metallic expandable tracheal stents in the management of inoperable malignant tracheal obstruction. Eur Arch Otorhinolaryngol 2012; 269: 247-53.

2. Finlayson GN, Brodsky JB. Anesthetic considerations for airway stenting in adult patients. Anesthesiol Clin 2008; 26: 281-91.

3. Pettignano R, Fortenberry JD, Heard ML, Labuz MD, Kesser KC, Tanner AJ, et al. Primary use of the venovenous approach for extracorporeal membrane oxygenation in pediatric acute respiratory failure. Pediatr Crit Care Med 2003; 4: 291-8.

4. Chauhan S, Subin S. Extracorporeal membrane oxygenation, an anesthesiologist's perspective: physiology and principles. Part 1 . Ann Card Anaesth 2011; 14: 218-29. 